PROCEEDINGS OF THE

AMERICAN MATHEMATICAL SOCIETY

Volume 133, Number 6, Pages 1805-1816

S 0002-9939(04)07727-5

Article electronically published on December 20, 2004

\title{
A NEW CRITERION FOR $k$-HYPONORMALITY VIA WEAK SUBNORMALITY
}

\author{
RAÚL E. CURTO, SANG HOON LEE, AND WOO YOUNG LEE
}

(Communicated by David R. Larson)

\begin{abstract}
In this article we obtain a criterion for $k$-hyponormality via weak subnormality. Using this criterion we recapture Spitkovskii's subnormality criterion and give a simple proof of the main result in Gu's preprint (2001), which describes a gap between $k$-hyponormality and $(k+1)$-hyponormality for Toeplitz operators. In addition, we notice that the minimal normal extension of a subnormal operator is exactly the inductive limit of its minimal partially normal extensions.
\end{abstract}

\section{INTRODUCTION}

Let $\mathcal{H}$ and $\mathcal{K}$ be separable complex Hilbert spaces, let $\mathcal{L}(\mathcal{H}, \mathcal{K})$ be the set of bounded linear operators from $\mathcal{H}$ to $\mathcal{K}$ and write $\mathcal{L}(\mathcal{H}):=\mathcal{L}(\mathcal{H}, \mathcal{H})$. An operator $T \in \mathcal{L}(\mathcal{H})$ is said to be normal if $T^{*} T=T T^{*}$, hyponormal if $T^{*} T \geq T T^{*}$, and subnormal if $T=\left.N\right|_{\mathcal{H}}$, where $N$ is normal on some Hilbert space $\mathcal{K} \supseteq \mathcal{H}$. Thus an operator $T$ is subnormal if and only if there exist operators $A$ and $B$ such that $\widehat{T}:=\left(\begin{array}{cc}T & A \\ 0 & B\end{array}\right)$ is normal, i.e.,

$$
\left\{\begin{array}{l}
{\left[T^{*}, T\right]:=T^{*} T-T T^{*}=A A^{*},} \\
A^{*} T=B A^{*} \\
{\left[B^{*}, B\right]+A^{*} A=0 .}
\end{array}\right.
$$

An operator $T \in \mathcal{L}(\mathcal{H})$ is said to be weakly subnormal ([CuL2]) if there exist operators $A \in \mathcal{L}\left(\mathcal{H}, \mathcal{H}^{\prime}\right)$ and $B \in \mathcal{L}\left(\mathcal{H}^{\prime}\right)$ such that the first two conditions in (0.1) hold: $\left[T^{*}, T\right]=A A^{*}$ and $A^{*} T=B T^{*}$, or equivalently, there is an extension $\widehat{T}$ of $T$ such that

$$
\widehat{T}^{*} \widehat{T} f=\widehat{T}^{*} f \text { for all } f \in \mathcal{H} \text {. }
$$

Received by the editors August 31, 2003 and, in revised form, February 23, 2004

2000 Mathematics Subject Classification. Primary 47B20, 47B35, 47A63; Secondary 47B37, 47B38, 47A05, 30D50.

Key words and phrases. k-hyponormal operators, subnormal operators, Toeplitz operators, unilateral weighted shifts, weak subnormality.

The work of the first-named author was partially supported by NSF research grants DMS9800931 and DMS-0099357.

The work of the third-named author was partially supported by KOSEF research project No. R01-2000-00003-0. 
The operator $\widehat{T}$ is said to be a partially normal extension (briefly, p.n.e.) of $T$. Note that the condition $(0.2)$ implies $\|\widehat{T} f\|=\left\|\widehat{T}^{*} f\right\|$ for all $f \in \mathcal{H}$, and that if (0.2) holds for all $f \in \mathcal{H} \oplus \mathcal{H}^{\prime}$, then $\widehat{T}$ becomes normal, so $T$ is in that case subnormal. We also say that $\widehat{T} \in \mathcal{L}(\mathcal{K})$ is a minimal partially normal extension (briefly, m.p.n.e.) of a weakly subnormal operator $T$ if $\mathcal{K}$ has no proper subspace containing $\mathcal{H}$ to which the restriction of $\widehat{T}$ is also a partially normal extension of $T$. It is known [CuL2, Lemma 2.5] that if $\widehat{T}$ is a partially normal extension of $T \in \mathcal{L}(\mathcal{H})$ on $\mathcal{K}$, then $\widehat{T}$ is minimal if and only if $\mathcal{K}=\bigvee\left\{\widehat{T}^{* k} h: h \in \mathcal{H}, k=0,1\right\}$ Clearly, subnormal $\Longrightarrow$ weakly subnormal $\Longrightarrow$ hyponormal; however, the converses are not true in general (cf. CuL2]).

On the other hand, the Bram-Halmos criterion for subnormality states that an operator $T$ is subnormal if and only if $\sum_{i, j}\left(T^{i} x_{j}, T^{j} x_{i}\right) \geq 0$ for all finite collections $x_{0}, x_{1}, \cdots, x_{k} \in \mathcal{H}$ ([Bra], [Con, II.1.9]). It is easy to see that this is equivalent to the following positivity test:

$$
\left(\begin{array}{cccc}
I & T^{*} & \ldots & T^{* k} \\
T & T^{*} T & \ldots & T^{* k} T \\
\vdots & \vdots & \ddots & \vdots \\
T^{k} & T^{*} T^{k} & \ldots & T^{* k} T^{k}
\end{array}\right) \geq 0 \quad(\text { all } k \geq 1)
$$

Condition (0.3) provides a measure of the gap between hyponormality and subnormality. In fact, the positivity condition $(0.3)$ for $k=1$ is equivalent to the hyponormality of $T$, while subnormality requires the validity of $(0.3)$ for all $k$. If we denote by $[A, B]:=A B-B A$ the commutator of two operators $A$ and $B$, and if we define $T$ to be $k$-hyponormal whenever the $k \times k$ operator matrix

$$
M_{k}(T):=\left(\left[T^{* j}, T^{i}\right]\right)_{i, j=1}^{k}
$$

is positive, or equivalently, the $(k+1) \times(k+1)$ operator matrix in $(0.3)$ is positive (via the operator version of Choleski's Algorithm), then the Bram-Halmos criterion can be rephrased as saying that $T$ is subnormal if and only if $T$ is $k$-hyponormal for every $k \geq 1$ ([CMX] $)$. The classes of $k$-hyponormal operators have been studied in an attempt to bridge the gap between subnormality and hyponormality ([Cu1], [Cu2, [CuF1], [CuF2], [CuF3], [CuL1], [CuL2], CuL3], [CMX], [DPY], [McCP]).

In this paper we obtain a new, different criterion for $k$-hyponormality via weak subnormality. Our criterion is sometimes more helpful because it avoids the potentially complicated verification of positivity needed for (0.4). Using this criterion we recapture Spitkovskii's subnormality criterion Spi and give a simple proof of the main result in $[\mathrm{Gu}]$, which describes a gap between $k$-hyponormality and $(k+1)$ hyponormality for Toeplitz operators.

\section{A NEW CRITERION FOR $k$-HYPONORMALITY}

Given a bounded sequence of positive numbers $\alpha: \alpha_{0}, \alpha_{1}, \cdots$ (called weights), the (unilateral) weighted shift $W_{\alpha}$ associated with $\alpha$ is the operator on $\ell^{2}\left(\mathbb{Z}_{+}\right)$defined by $W_{\alpha} e_{n}:=\alpha_{n} e_{n+1}$ for all $n \geq 0$, where $\left\{e_{n}\right\}_{n=0}^{\infty}$ is the canonical orthonormal basis for $\ell^{2}$. It is straightforward to check that $W_{\alpha}$ can never be normal, and that $W_{\alpha}$ is hyponormal if and only if $\alpha_{n} \leq \alpha_{n+1}$ for all $n \geq 0$. 
In 1966, Stampfli [Sta] explicitly exhibited for a subnormal weighted shift $A_{0}$ its minimal normal extension

$$
N:=\left(\begin{array}{cccc}
A_{0} & B_{1} & & 0 \\
& A_{1} & B_{2} & \\
& & A_{2} & \ddots \\
& & & \ddots
\end{array}\right),
$$

where $A_{n}$ is a weighted shift with weights $\left\{a_{0}^{(n)}, a_{1}^{(n)}, \cdots\right\}, B_{n}:=\operatorname{diag}\left\{b_{0}^{(n)}, b_{1}^{(n)}, \cdots\right\}$ and these entries satisfy:

(I) $\left(a_{j}^{(n)}\right)^{2}-\left(a_{j-1}^{(n)}\right)^{2}+\left(b_{j}^{(n)}\right)^{2} \geq 0\left(b_{j}^{(0)}=0\right.$ for all $\left.j\right)$;

(II) $b_{j}^{(n)}=0 \Longrightarrow b_{j+1}^{(n)}=0$;

(III) there exists a constant $M$ such that $\left|a_{j}^{(n)}\right| \leq M$ and $\left|b_{j}^{(n)}\right| \leq M$ for $n=$ $0,1, \cdots$ and $j=0,1, \cdots$, where

$$
b_{j}^{(n+1)}:=\left[\left(a_{j}^{(n)}\right)^{2}-\left(a_{j-1}^{(n)}\right)^{2}+\left(b_{j}^{(n)}\right)^{2}\right]^{\frac{1}{2}} \quad \text { and } \quad a_{j}^{(n+1)}:=a_{j}^{(n)} \frac{b_{j+1}^{(n+1)}}{b_{j}^{(n+1)}}
$$

(if $b_{j_{0}}^{(n)}=0$, then $a_{j_{0}}^{(n)}$ is taken to be 0 ).

On the other hand, in 1982, I. Spitkovskii [Spi] gave the following subnormality criterion for arbitrary operators. (In 1985, J. Ma and S. Zhou MaZ] independently proved the same result.)

Theorem 1 ([Spi $)$. Let $A_{0} \in \mathcal{L}\left(\mathcal{H}_{0}\right)$, where $\mathcal{H}_{0}$ is a separable complex Hilbert space. Then $A_{0}$ is subnormal if and only if there exists $M>0$ such that for every $n>0$ the following properties hold:

(I') $D_{n} \geq 0$;

(II') $A_{n-1}\left(\operatorname{Ker} D_{n-1}\right) \subseteq \operatorname{Ker} D_{n-1}(n \geq 1)$;

(III') there exists a constant $M$ such that $\left\|A_{n}\right\|,\left\|D_{n}\right\| \leq M$, where

$$
\begin{aligned}
D_{0} & :=\left[A_{0}^{*}, A_{0}\right], \quad D_{n+1}:=\left.D_{n}\right|_{\mathcal{H}_{n+1}}+\left[A_{n+1}^{*}, A_{n+1}\right], \quad \mathcal{H}_{n+1}:=\overline{\operatorname{Ran}\left(D_{n}\right)}, \\
& \text { and } A_{n+1} \text { denotes the bounded extension of } D_{n}^{\frac{1}{2}} A_{n} D_{n}^{-\frac{1}{2}} \text { to } \overline{\operatorname{Ran}\left(D_{n}\right)} \\
& \left(=\mathcal{H}_{n+1}\right) \text { from } \operatorname{Ran}\left(D_{n}\right) .
\end{aligned}
$$

In this case, the minimal normal extension of $A_{0}$ is given by the operator $N$ in (0.5) with $D_{n-1}^{\frac{1}{2}}$ in place of $B_{n}$.

In 1988, P. Fan Fan noticed that if $A_{0}$ is a weighted shift, then (I) and (I'), (II) and $\left(\mathrm{II}^{\prime}\right)$, and (III) and (III') are equivalent, respectively. Consequently, Theorem 1 is a natural generalization of Stampfli's subnormality criterion for weighted shifts.

We will now discuss analogues of the preceding results for $k$-hyponormal operators. It was shown in CuL2 that every 2-hyponormal weighted shift is weakly subnormal, but whether the same implication holds for arbitrary operators was left open. Very recently, R. Curto, I. B. Jung and S. S. Park CJP showed that every 2-hyponormal operator is indeed weakly subnormal. This follows from a special case of a more general result:

Lemma 2 ([CJP Theorems 2.7 and 3.2]). (i) If $T \in \mathcal{L}(\mathcal{H})$ is 2-hyponormal, then $\left.\left[T^{*}, T\right]^{\frac{1}{2}} T\left[T^{*}, T\right]^{-\frac{1}{2}}\right|_{\operatorname{Ran}\left[T^{*}, T\right]}$ is bounded; 
(ii) $T \in \mathcal{L}(\mathcal{H})$ is $(k+1)$-hyponormal if and only if $T$ is weakly subnormal and $\widehat{T}:=$ m.p.n.e. $(T)$ is k-hyponormal.

In particular, it was shown in CuL2, Lemma 2.8] that if $A_{0} \in \mathcal{L}\left(\mathcal{H}_{0}\right)$ is weakly subnormal, then the minimal partially normal extension of $A_{0}$ can be obtained as

$$
\widehat{A_{0}}=\left(\begin{array}{cc}
A_{0} & D_{0} \\
0 & A_{1}
\end{array}\right): \mathcal{H}_{0} \oplus \mathcal{H}_{1} \rightarrow \mathcal{H}_{0} \oplus \mathcal{H}_{1},
$$

where $\mathcal{H}_{1} \equiv \overline{\operatorname{Ran}\left[A_{0}^{*}, A_{0}\right]}$ and $D_{0}$ is the restriction of $\left[A_{0}^{*}, A_{0}\right]^{\frac{1}{2}}$ to $\mathcal{H}_{1}$ satisfying $D_{0} A_{0}=A_{1} D_{0}$. Note that

$$
\bigvee\left\{{\widehat{A_{0}}}^{* k} h_{0}: h_{0} \in \mathcal{H}_{0}, k=0,1\right\}=\mathcal{H}_{0} \oplus \mathcal{H}_{1} .
$$

By using Lemma 2(ii) inductively, we can see that if $A_{0}$ is $k$-hyponormal, then we can define

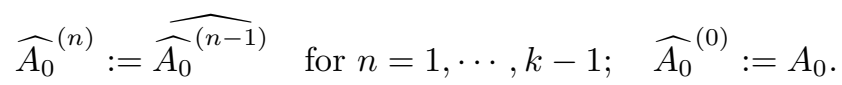

Our criterion on $k$-hyponormality now follows:

Theorem 3. An operator $A_{0} \in \mathcal{L}\left(\mathcal{H}_{0}\right)$ is $k$-hyponormal if and only if the following three conditions hold for all $n$ such that $0 \leq n \leq k-1$ :

( $\left.\mathrm{I}_{n}\right) D_{n} \geq 0$;

(II $\left.{ }_{n}\right) A_{n-1}\left(\operatorname{Ker} D_{n-1}\right) \subseteq \operatorname{Ker} D_{n-1}(n \geq 1)$;

$\left.\left(\mathrm{III}_{n}\right) D_{n-1}^{\frac{1}{2}} A_{n-1} D_{n-1}^{-\frac{1}{2}}\right|_{\operatorname{Ran}\left(D_{n-1}\right)}(n \geq 1)$ is bounded, where

$$
D_{0}:=\left[A_{0}^{*}, A_{0}\right], \quad D_{n+1}:=\left.D_{n}\right|_{\mathcal{H}_{n+1}}+\left[A_{n+1}^{*}, A_{n+1}\right], \quad \mathcal{H}_{n+1}:=\overline{\operatorname{Ran}\left(D_{n}\right)}
$$

and $A_{n+1}$ denotes the bounded extension of $D_{n}^{\frac{1}{2}} A_{n} D_{n}^{-\frac{1}{2}}$ to $\overline{\operatorname{Ran}\left(D_{n}\right)}\left(=\mathcal{H}_{n+1}\right)$ from $\operatorname{Ran}\left(D_{n}\right)$.

Proof. Suppose $A_{0}$ is $k$-hyponormal. We now use induction on $k$. If $k=2$, then $A_{0}$ is 2-hyponormal, and so $D_{0}:=\left[A_{0}^{*}, A_{0}\right] \geq 0$. By Lemma $2(\mathrm{i}),\left.D_{0}^{\frac{1}{2}} A_{0} D_{0}^{-\frac{1}{2}}\right|_{\operatorname{Ran}\left(D_{0}\right)}$ is bounded. Let $A_{1}$ be the bounded extension of $D_{0}^{\frac{1}{2}} A_{0} D_{0}^{-\frac{1}{2}}$ from $\operatorname{Ran}\left(D_{0}\right)$ to $\mathcal{H}_{1}:=\overline{\operatorname{Ran}\left(D_{0}\right)}$ and $D_{1}:=\left.D_{0}\right|_{\mathcal{H}_{1}}+\left[A_{1}^{*}, A_{1}\right]$. Writing $\widehat{A_{0}}:=\left(\begin{array}{cc}A_{0} & D_{0}^{\frac{1}{2}} \\ 0 & A_{1}\end{array}\right)$, we have $\widehat{A_{0}}=$ m.p.n.e. $\left(A_{0}\right)$, which is hyponormal by Lemma 2(ii). Thus

$$
\left[{\widehat{A_{0}}}^{*}, \widehat{A_{0}}\right]=\left(\begin{array}{cc}
0 & 0 \\
0 & \left.D_{0}\right|_{\mathcal{H}_{1}}+\left[A_{1}^{*}, A_{1}\right]
\end{array}\right) \geq 0,
$$

and hence $D_{1} \geq 0$. Also by $\mathrm{CuL2}$, Lemma 2.2], $A_{0}\left(\operatorname{Ker} D_{0}\right) \subseteq \operatorname{Ker} D_{0}$ whenever $A_{0}$ is 2-hyponormal. Thus $\left(\mathrm{I}_{n}\right),\left(\mathrm{II}_{n}\right)$, and $\left(\mathrm{III}_{n}\right)$ hold for $n=0,1$. Assume now that if $A_{0}$ is $k$-hyponormal, then $\left(\mathrm{I}_{n}\right),\left(\mathrm{II}_{n}\right)$ and $\left(\mathrm{III}_{n}\right)$ hold for all $0 \leq n \leq k-1$. Suppose $A_{0}$ is $(k+1)$-hyponormal. We must show that $\left(\mathrm{I}_{n}\right),\left(\mathrm{II}_{n}\right)$ and $\left(\mathrm{III}_{n}\right)$ hold for $n=k$. Define

$$
S:=\left(\begin{array}{ccccc}
A_{0} & D_{0}^{\frac{1}{2}} & & & 0 \\
& A_{1} & D_{1}^{\frac{1}{2}} & & \\
& & \ddots & \ddots & \\
& & & \ddots & D_{k-2}^{\frac{1}{2}} \\
0 & & & & A_{k-1}
\end{array}\right): \bigoplus_{i=0}^{k-1} \mathcal{H}_{i} \longrightarrow \bigoplus_{i=0}^{k-1} \mathcal{H}_{i} .
$$


By our inductive assumption, $D_{k-1} \geq 0$. Writing $\widehat{T}^{(n)}:=$ m.p.n.e. $\left(\widehat{T}^{(n-1)}\right)$ when it exists, we can see by our assumption that $S={\widehat{A_{0}}}^{(k-1)}$ : indeed, if

$$
S_{l}:=\left(\begin{array}{ccccc}
A_{0} & D_{0}^{\frac{1}{2}} & & & 0 \\
& A_{1} & D_{1}^{\frac{1}{2}} & & \\
& & \ddots & \ddots & \\
& & & \ddots & D_{l-2}^{\frac{1}{2}} \\
0 & & & & A_{l-1}
\end{array}\right),
$$

then since by assumption $\left[S_{l}^{*}, S_{l}\right]=0 \oplus D_{l}$ and $A_{l}=\left.D_{l-1}^{\frac{1}{2}} A_{l-1} D_{l-1}^{-\frac{1}{2}}\right|_{\operatorname{Ran}\left(D_{l-1}\right)}$, it follows that $S_{l}$ is the minimal partially normal extension of $S_{l-1}(1 \leq l \leq k-1)$. But since by our assumption $A_{0}$ is $(k+1)$-hyponormal, it follows from Lemma 2(ii) that $S$ is 2 -hyponormal. Thus by Lemma $2(\mathrm{i}),\left.\left[S^{*}, S\right]^{\frac{1}{2}} S\left[S^{*}, S\right]^{-\frac{1}{2}}\right|_{\operatorname{Ran}\left(\left[S^{*}, S\right]\right)}$ is bounded, which says that $\left.D_{k-1}^{\frac{1}{2}} A_{k-1} D_{k-1}^{-\frac{1}{2}}\right|_{\operatorname{Ran}\left(D_{k-1}\right)}$ is bounded, proving (III ${ }_{n}$ ) for $n=k$. Observe that $A_{k}, \mathcal{H}_{k}$ and $D_{k}$ are well-defined. Writing $\widehat{S}:=\left(\begin{array}{cc}S & D_{k-1}^{\frac{1}{2}} \\ 0 & A_{k}\end{array}\right)$, we can see that $\widehat{S}=$ m.p.n.e. $(S)$, which is hyponormal, again by Lemma 2(ii). Thus, since $\left[\widehat{S}^{*}, \widehat{S}\right]=\left(\begin{array}{cc}0 & 0 \\ 0 & D_{k}\end{array}\right) \geq 0$, we have $D_{k} \geq 0$, proving $\left(\mathrm{I}_{n}\right)$ for $n=k$. On the other hand, since $S$ is 2-hyponormal, it follows that $S\left(\operatorname{Ker}\left[S^{*}, S\right]\right) \subseteq \operatorname{Ker}\left[S^{*}, S\right]$. Since $\left[S^{*}, S\right]=\left(\begin{array}{cc}0 & 0 \\ 0 & D_{k-1}\end{array}\right)$, we have $\operatorname{Ker}\left[S^{*}, S\right]=\bigoplus_{i=0}^{k-2} \mathcal{H}_{i} \oplus \operatorname{Ker}\left(D_{k-1}\right)$. Thus, since

$$
\left(\begin{array}{ccccc}
A_{0} & D_{0}^{\frac{1}{2}} & & & 0 \\
& A_{1} & D_{1}^{\frac{1}{2}} & & \\
& & \ddots & \ddots & \\
& & & \ddots & D_{k-2}^{\frac{1}{2}} \\
0 & & & & A_{k-1}
\end{array}\right)\left(\begin{array}{c}
\mathcal{H}_{0} \\
\mathcal{H}_{1} \\
\vdots \\
\mathcal{H}_{k-2} \\
\operatorname{Ker}\left(D_{k-1}\right)
\end{array}\right) \subseteq\left(\begin{array}{c}
\mathcal{H}_{0} \\
\mathcal{H}_{1} \\
\vdots \\
\mathcal{H}_{k-2} \\
\operatorname{Ker}\left(D_{k-1}\right)
\end{array}\right),
$$

we must have that $A_{k-1}\left(\operatorname{Ker}\left(D_{k-1}\right)\right) \subseteq \operatorname{Ker}\left(D_{k-1}\right)$, proving $\left(\mathrm{II}_{n}\right)$ for $n=k$. This proves the necessity condition.

Toward sufficiency, suppose that conditions $\left(\mathrm{I}_{n}\right),\left(\mathrm{II}_{n}\right)$ and $\left(\mathrm{III}_{n}\right)$ hold for all $n$ such that $0 \leq n \leq k-1$. Define

$$
S_{n}:=\left(\begin{array}{ccccc}
A_{0} & D_{0}^{\frac{1}{2}} & & & 0 \\
& A_{1} & D_{1}^{\frac{1}{2}} & & \\
& & \ddots & \ddots & \\
& & & \ddots & D_{n-2}^{\frac{1}{2}} \\
0 & & & & A_{n-1}
\end{array}\right) \quad(1 \leq n \leq k-1) .
$$

Then $S_{k-2}$ is weakly subnormal and $S_{k-1}=$ m.p.n.e. $\left(S_{k-2}\right)$. Since, by assumption, $D_{k-1} \geq 0$, we have $\left[S_{k-1}^{*}, S_{k-1}\right]=\left(\begin{array}{cc}0 & 0 \\ 0 & D_{k-1}\end{array}\right) \geq 0$. It thus follows from Lemma 2(ii) that $S_{k-2}$ is 2-hyponormal. Note that $S_{n}=$ m.p.n.e. $\left(S_{n-1}\right)$ for $n=1, \cdots, k-1$ $\left(S_{0}:=A_{0}\right)$. Thus, again by Lemma $2(\mathrm{ii}), S_{k-3}$ is 3 -hyponormal. Now repeating 
this argument, we can conclude that $S_{0} \equiv A_{0}$ is $k$-hyponormal. This completes the proof.

We now present a strengthened version of Spitkovskii's subnormality criterion.

Corollary 4. An operator $A_{0} \in \mathcal{L}\left(\mathcal{H}_{0}\right)$ is subnormal if and only if the conditions $\left(\mathrm{I}_{n}\right),\left(\mathrm{II}_{n}\right)$, and $\left(\mathrm{III}_{n}\right)$ hold for all $n \geq 0$. In this case, the minimal normal extension $N$ of $A_{0}$ is given by

$$
N=\left(\begin{array}{cccc}
A_{0} & D_{0}^{\frac{1}{2}} & & 0 \\
& A_{1} & D_{1}^{\frac{1}{2}} & \\
& & A_{2} & \ddots \\
0 & & & \ddots
\end{array}\right): \bigoplus_{i=0}^{\infty} \mathcal{H}_{i} \rightarrow \bigoplus_{i=0}^{\infty} \mathcal{H}_{i} .
$$

Proof. The first assertion follows from Theorem 3 together with the fact that $A_{0}$ is subnormal if and only if $A_{0}$ is $k$-hyponormal for all $k \geq 1$. Toward the second assertion, observe by a straightforward calculation that $N^{*} N=N N^{*}$, i.e., $N$ is a (possibly unbounded) normal operator. For the boundedness of $N$, observe that

(i)

$$
\left(\begin{array}{ccccc}
A_{0} & D_{0}^{\frac{1}{2}} & & & 0 \\
& A_{1} & D_{1}^{\frac{1}{2}} & & \\
& & \ddots & \ddots & \\
& & & \ddots & D_{n-1}^{\frac{1}{2}} \\
0 & & & & A_{n}
\end{array}\right)={\widehat{A_{0}}}^{(n)}
$$

(ii) $\left\|A_{0}\right\|=\left\|\widehat{A}_{0}^{(n)}\right\|$ for all $n \geq 1$ (see [CJP, Corollary 3.3]).

Thus we can see that $\left\|A_{n}\right\|,\left\|D_{n-1}^{\frac{1}{2}}\right\| \leq\left\|A_{0}\right\|$ for all $n \geq 1$. Therefore, evidently, $N$ is bounded. For the minimality of $N$ we must show that

$$
\bigoplus_{i=0}^{\infty} \mathcal{H}_{i}=\bigvee\left\{N^{* k} h_{0}: h_{0} \in \mathcal{H}_{0}, k \geq 0\right\}
$$

To show this it will suffice to prove that

$$
\bigoplus_{i=0}^{n} \mathcal{H}_{i}=\bigvee\left\{\left({\widehat{A_{0}}}^{(n)}\right)^{* k} h_{0}: h_{0} \in \mathcal{H}_{0}, k=0,1, \cdots, n\right\} \quad \text { for all } n \geq 1
$$

We use induction on $n$. Since $\widehat{A_{0}}=$ m.p.n.e. $\left(A_{0}\right)$ it follows from $(2.1)$ that

$$
\mathcal{H}_{0} \oplus \mathcal{H}_{1}=\bigvee\left\{{\widehat{A_{0}}}^{* k} h_{0}: h_{0} \in \mathcal{H}_{0}, k=0,1\right\}
$$

which proves (4.2) for $n=1$. We assume that (4.2) holds for $n=m$. Note that

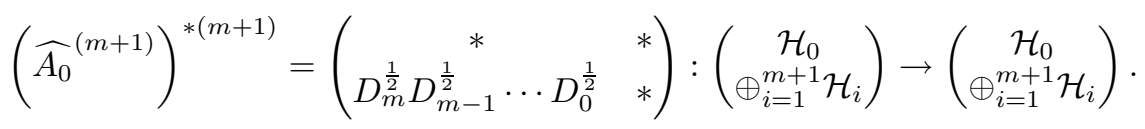


Thus, we have

$$
\begin{aligned}
& \bigvee\left\{\left(\widehat{A}_{0}^{(m+1)}\right)^{* k} h_{0}: h_{0} \in \mathcal{H}_{0}, k=0,1, \cdots, m+1\right\} \\
= & \left.\bigvee\left\{\left\{\widehat{A}_{0}^{(m+1)}\right)^{* k} h_{0}: h_{0} \in \mathcal{H}_{0}, k=0,1, \cdots, m\right\},\left(\widehat{A}_{0}^{(m+1)}\right)^{*(m+1)}\left(\mathcal{H}_{0}\right)\right\} \\
= & \bigvee\left\{\bigoplus_{i=0}^{m} \mathcal{H}_{i},\left(\widehat{A}_{0}^{(m+1)}\right)^{*(m+1)}\left(\mathcal{H}_{0}\right)\right\} \\
= & \bigvee\left\{\left(\bigoplus_{i=0}^{m} \mathcal{H}_{i}\right) \oplus D_{m}^{\frac{1}{2}} D_{m-1}^{\frac{1}{2}} \cdots D_{0}^{\frac{1}{2}}\left(\mathcal{H}_{0}\right)\right\}=\bigoplus_{i=0}^{m+1} \mathcal{H}_{i},
\end{aligned}
$$

which proves (4.2) for $n=m+1$. This completes the proof.

It is interesting to note that Corollary 4 is a strengthened version of Spitkovskii's criterion in the sense that Spitkovskii's criterion requires the uniform boundedness of $\left\{A_{n}\right\}$ and $\left\{D_{n}\right\}$ for subnormality of $A_{0}$, while our criterion drops this condition. In fact, the uniform boundedness condition follows automatically from the construction of the minimal partially normal extension via weak subnormality as we saw in the proof of Corollary 4 . This is an advantage of using weak subnormality.

\section{Gaps Between $k$-HYPONORMALity AND SUbNoRmality FOR TOEPLITZ OPERATORS}

Recall that the Hilbert space $L^{2}(\mathbb{T})$ has a canonical orthonormal basis given by the trigonometric functions $e_{n}(z)=z^{n}$, for all $n \in \mathbb{Z}$, and that the Hardy space $H^{2}(\mathbb{T})$ is the closed linear span of $\left\{e_{n}: n=0,1, \cdots\right\}$. An element $f \in L^{2}(\mathbb{T})$ is said to be analytic if $f \in H^{2}(\mathbb{T})$, and co-analytic if $f \in L^{2}(\mathbb{T}) \ominus H^{2}(\mathbb{T})$. If $P$ denotes the orthogonal projection from $L^{2}(\mathbb{T})$ to $H^{2}(\mathbb{T})$, then for every $\varphi \in L^{\infty}(\mathbb{T})$ the operator $T_{\varphi}$ on $H^{2}(\mathbb{T})$ defined by

$$
T_{\varphi} g:=P(\varphi g) \quad\left(g \in H^{2}(\mathbb{T})\right)
$$

is called the Toeplitz operator with symbol $\varphi$.

It was recently shown in CLL that there exists a gap between 2-hyponormality and subnormality for Toeplitz operators. Subsequently, C. Gu Gu gave a more general result: there exists a gap between $k$-hyponormality and $(k+1)$-hyponormality for Toeplitz operators for each $k \geq 1$.

Theorem $5([\mathrm{Gu}$, Theorem 3.5]). Let $0<\alpha<1$ and let $\psi$ be the conformal map of the unit disk onto the interior of the ellipse with vertices $\pm(1+\alpha) i$ and passing through $\pm(1-\alpha)$. Let $\varphi=\psi+\lambda \bar{\psi}$ and let $T_{\varphi}$ be the corresponding Toeplitz operator on $H^{2}$. Then $T_{\varphi}$ is k-hyponormal if and only if $\lambda$ is in the circle $\left|z-\frac{\alpha\left(1-\alpha^{2 j}\right)}{1-\alpha^{2 j+2}}\right|=$ $\frac{\alpha^{j}\left(1-\alpha^{2}\right)}{1-\alpha^{2 j+2}}$ for $j=0,1, \cdots, k-2$ or in the closed disk $\left|z-\frac{\alpha\left(1-\alpha^{2(k-1)}\right)}{1-\alpha^{2 k}}\right| \leq \frac{\alpha^{k-1}\left(1-\alpha^{2}\right)}{1-\alpha^{2 k}}$.

Gu's proof of Theorem 5 relies on intricate and explicit computations using a special case of Smul'jan's Theorem [Smu]. We here give a simple proof using our criterion (Theorem 3). This illustrates that our criterion on $k$-hyponormality is more effective than the positivity conditions (0.3) or (0.4). 
For $0<\alpha<1$, let $T \equiv W_{\beta}$ be the weighted shift with weight sequence $\beta=$ $\left\{\beta_{n}\right\}_{n=0}^{\infty}$, where (cf. [Cow2, Proposition 9])

$$
\beta_{n}:=\left(\sum_{j=0}^{n} \alpha^{2 j}\right)^{\frac{1}{2}} \text { for } n=0,1, \cdots
$$

Let $D$ be the diagonal operator, $D=\operatorname{diag}\left(\alpha^{n}\right)$, and let $S_{\lambda} \equiv T+\lambda T^{*}(\lambda \in \mathbb{C})$. Then we have that

$$
\left[T^{*}, T\right]=D^{2}=\operatorname{diag}\left(\alpha^{2 n}\right) \quad \text { and } \quad\left[S_{\lambda}^{*}, S_{\lambda}\right]=\left(1-|\lambda|^{2}\right)\left[T^{*}, T\right]=\left(1-|\lambda|^{2}\right) D^{2} .
$$

Define

It follows that $A_{0}=S_{\lambda}$ and

$$
A_{l}:=\alpha^{l} T+\frac{\lambda}{\alpha^{l}} T^{*} \quad(l=0, \pm 1, \pm 2, \cdots) .
$$

$$
D A_{l}=A_{l+1} D \quad \text { and } \quad A_{l}^{*} D=D A_{l+1}^{*} \quad(l=0, \pm 1, \pm 2, \cdots) .
$$

The following theorem is the essence of C. Gu's argument [Gu], which consists of a complicated computation. By contrast our proof is shorter and more transparent.

Theorem 6 ([Gu, Theorem 3.3]). Let $0<\alpha<1$ and $T \equiv W_{\beta}$ be the weighted shift with weight sequence $\beta=\left\{\beta_{n}\right\}_{n=0}^{\infty}$, where

$$
\beta_{n}=\left(\sum_{j=0}^{n} \alpha^{2 j}\right)^{\frac{1}{2}} \text { for } n=0,1, \cdots .
$$

Then $A_{0}:=T+\lambda T^{*}$ is $k$-hyponormal if and only if $|\lambda| \leq \alpha^{k-1}$ or $|\lambda|=\alpha^{j}$ for some $j=0,1, \cdots, k-2$.

Proof. Observe that

$$
\begin{aligned}
{\left[A_{l}^{*}, A_{l}\right] } & =\left[\alpha^{l} T^{*}+\frac{\bar{\lambda}}{\alpha^{l}} T, \alpha^{l} T+\frac{\lambda}{\alpha^{l}} T^{*}\right] \\
& =\alpha^{2 l}\left[T^{*}, T\right]-\frac{|\lambda|^{2}}{\alpha^{2 l}}\left[T^{*}, T\right]=\left(\alpha^{2 l}-\frac{|\lambda|^{2}}{\alpha^{2 l}}\right) D^{2} .
\end{aligned}
$$

Since Ker $D=\{0\}$ and $D A_{n}=A_{n+1} D$, it follows that $\mathcal{H}_{n}=\mathcal{H}$ for all $n$; if we use $A_{l}$ for the operator $A_{n}$ in Theorem 3 then we have, by (6.1) and the definition of $D_{j}$, that

$$
\begin{aligned}
D_{j} & =D_{j-1}+\left[A_{j}^{*}, A_{j}\right]=D_{j-2}+\left[A_{j-1}^{*}, A_{j-1}\right]+\left[A_{j}^{*}, A_{j}\right]=\cdots \\
& =\left[A_{0}^{*}, A_{0}\right]+\left[A_{1}^{*}, A_{1}\right]+\cdots+\left[A_{j}^{*}, A_{j}\right]=\left(1-|\lambda|^{2}\right) D^{2}+\cdots+\left(\alpha^{2 j}-\frac{|\lambda|^{2}}{\alpha^{2 j}}\right) D^{2} \\
& =\left(\frac{1-\alpha^{2(j+1)}}{1-\alpha^{2}}\right)\left(1-\frac{|\lambda|^{2}}{\alpha^{2 j}}\right) D^{2} .
\end{aligned}
$$

By Theorem $3, A_{0}$ is $k$-hyponormal if and only if $D_{k-1} \geq 0$ or $D_{j}=0$ for some $j$ such that $0 \leq j \leq k-2$ (in this case $A_{0}$ is subnormal). Note that $D_{j}=0$ if and only if $|\lambda|=\alpha^{j}$. On the other hand, if $D_{j}>0$ for $j=0,1, \cdots, k-2$, then

$$
D_{k-1}=\left(\frac{1-\alpha^{2 k}}{1-\alpha^{2}}\right)\left(1-\frac{|\lambda|^{2}}{\alpha^{2(k-1)}}\right) D^{2} \geq 0
$$

if and only if $|\lambda| \leq \alpha^{k-1}$. Therefore $A_{0}$ is $k$-hyponormal if and only if $|\lambda| \leq \alpha^{k-1}$ or $|\lambda|=\alpha^{j}$ for some $j, j=0,1, \cdots, k-2$. 
We are ready for:

Proof of Theorem 5. It was shown in $[\mathrm{CoL}]$ that $T_{\psi+\alpha \bar{\psi}}$ is unitarily equivalent to $\left(1-\alpha^{2}\right)^{\frac{3}{2}} T$, where $T$ is the weighted shift in Theorem 6 . Thus $T_{\psi}$ is unitarily equivalent to $\left(1-\alpha^{2}\right)^{\frac{1}{2}}\left(T-\alpha T^{*}\right)$, so $T_{\varphi}$ is unitarily equivalent to

$$
\left(1-\alpha^{2}\right)^{\frac{1}{2}}(1-\lambda \alpha)\left(T+\frac{\lambda-\alpha}{1-\lambda \alpha} T^{*}\right) \quad(\text { cf. [Cow1, Theorem 2.4]). }
$$

Applying Theorem 6 with $\frac{\lambda-\alpha}{1-\lambda \alpha}$ in place of $\lambda$, we have that for $k=0,1,2, \cdots$,

$$
\begin{aligned}
\left|\frac{\lambda-\alpha}{1-\lambda \alpha}\right| \leq \alpha^{k} & \Longleftrightarrow|\lambda-\alpha|^{2} \leq \alpha^{2 k}|1-\lambda \alpha|^{2} \\
& \Longleftrightarrow|\lambda|^{2}-\frac{\alpha\left(1-\alpha^{2 k}\right)}{1-\alpha^{2 k+2}}(\lambda+\bar{\lambda})+\frac{\alpha^{2}-\alpha^{2 k}}{1-\alpha^{2 k+2}} \leq 0 \\
& \Longleftrightarrow\left|\lambda-\frac{\alpha\left(1-\alpha^{2 k}\right)}{1-\alpha^{2 k+2}}\right| \leq \frac{\alpha^{k}\left(1-\alpha^{2}\right)}{1-\alpha^{2 k+2}} .
\end{aligned}
$$

This completes the proof.

\section{3. $k$-HYPONORMALITY OF WEIGHTED SHIFTS}

If $A_{0}$ is a weighted shift, then by Theorem 3 and the remarks following Theorem 1 we know that the following are equivalent:

(i) $A_{0}$ is $k$-hyponormal;

(ii) conditions (I), (II), (III), given right after (0.5), hold for all $n, 0 \leq n \leq k-1$.

In [Cu1, Theorem 4], it was shown that the $k$-hyponormality for a weighted shift $W_{\alpha}$ with $\alpha \equiv\left\{\alpha_{n}\right\}_{n=0}^{\infty}$ can be tested by the positivity of a $(k+1) \times(k+1)$ Hankel matrix $A(n ; k)$ built in terms of the moments $\gamma_{n}$ of $W_{\alpha}$, where

$$
\beta_{0}:=1, \quad \beta_{n+1}:=\alpha_{n} \beta_{n}(n \geq 0), \quad \gamma_{n}:=\beta_{n}^{2}(n \geq 0)
$$

and

$$
A(n ; k):=\left(\begin{array}{cccc}
\gamma_{n} & \gamma_{n+1} & \ldots & \gamma_{n+k} \\
\gamma_{n+1} & \gamma_{n+2} & \ldots & \gamma_{n+k+1} \\
\vdots & \vdots & & \vdots \\
\gamma_{n+k} & \gamma_{n+k+1} & \ldots & \gamma_{n+2 k}
\end{array}\right) \quad(n \geq 0)
$$

Thus we have:

Corollary 7 ( $k$-Hyponormality of weighted shifts). Let $A_{0}$ be a weighted shift with weight sequence $\left\{\alpha_{n}\right\}_{n=0}^{\infty}$. Then the following are equivalent:

(i) $A_{0}$ is k-hyponormal;

(ii) $A(n ; k) \geq 0$ for all $n \geq 0$;

(iii) conditions (I), (II) and (III) hold for all $n, 0 \leq n \leq k-1$.

Sometimes the equivalence (i) $\Leftrightarrow$ (iii) is more helpful than the equivalence (i) $\Leftrightarrow$ (ii). The following example illustrates this fact.

Example 8. For $x>0$, let $T_{x}$ be the weighted shift whose weight sequence is given by

$$
\alpha_{0}:=x, \quad \alpha_{n}=\sqrt{\frac{n+1}{n+2}} \quad(n \geq 1) .
$$


Then $T_{x}$ is $k$-hyponormal if and only if $x \leq \frac{k+1}{\sqrt{2 k(k+2)}}$. In particular, $T_{x}$ is subnormal

if and only if $x \leq \sqrt{\frac{1}{2}}$; that is, the Bergman shift is extremal amongst all subnormal weighted shifts of the form $T_{x}$.

Proof. We use the equivalence (i) $\Leftrightarrow$ (iii) in Corollary 7 . Write $a_{j}^{(0)}:=\alpha_{j}(j \geq 0)$. First, observe that

$$
\begin{array}{r}
T_{x} \text { is } k \text {-hyponormal } \Longleftrightarrow\left(b_{j}^{(k)}\right)^{2}:=\left(a_{j}^{(k-1)}\right)^{2}-\left(a_{j-1}^{(k-1)}\right)^{2}+\left(b_{j}^{(k-1)}\right)^{2} \geq 0 \\
\text { for all } j \geq 0 .
\end{array}
$$

Since $T_{x}$ has, beginning with $n=1$, a Bergman tail, it follows that

$$
T_{x} \text { is } k \text {-hyponormal } \Longleftrightarrow\left(b_{k}^{(k)}\right)^{2}:=\left(a_{k}^{(k-1)}\right)^{2}-\left(a_{k-1}^{(k-1)}\right)^{2}+\left(b_{k}^{(k-1)}\right)^{2} \geq 0 .
$$

We now claim that

$$
\left(b_{k}^{(k)}\right)^{2}=\frac{k}{2(2 k+1)} \cdot \frac{(k+1)^{2}-2 k(k+2) x^{2}}{k^{2}-2(k-1)(k+1) x^{2}} .
$$

Note that for $j \geq k+1$,

$$
\left(b_{j}^{(k)}\right)^{2}=\frac{k^{2}}{(j+k)(j+k+1)} \quad \text { and } \quad\left(a_{j}^{(k)}\right)^{2}=\frac{(j+1)^{2}}{(j+k+1)(j+k+2)} .
$$

For (8.1) we use induction on $k$. If $k=1$, then $\left(b_{1}^{(1)}\right)^{2}=\left(a_{1}^{(0)}\right)^{2}-\left(a_{0}^{(0)}\right)^{2}=\frac{2}{3}-x^{2}$, so (8.1) holds for $k=1$. Suppose that (8.1) holds for $k$. Then by (8.2),

$$
\begin{aligned}
\left(b_{k+1}^{(k+1)}\right)^{2} & =\left(a_{k+1}^{(k)}\right)^{2}-\left(a_{k}^{(k)}\right)^{2}+\left(b_{k+1}^{(k)}\right)^{2} \\
& =\left(a_{k+1}^{(k)}\right)^{2}-\left(a_{k}^{(k-1)}\right)^{2} \frac{\left(b_{k+1}^{(k)}\right)^{2}}{\left(b_{k}^{(k)}\right)^{2}}+\left(b_{k+1}^{(k)}\right)^{2} \\
& =\left(a_{k+1}^{(k)}\right)^{2}-\left(b_{k+1}^{(k)}\right)^{2}\left(\frac{\left(a_{k}^{(k-1)}\right)^{2}-\left(b_{k}^{(k)}\right)^{2}}{\left(b_{k}^{(k)}\right)^{2}}\right) \\
& =\left(a_{k+1}^{(k)}\right)^{2}-\left(b_{k+1}^{(k)}\right)^{2}\left(\frac{2(2 k+1) x^{2}}{k^{2}\left\{(k+1)^{2}-2 k(k+2) x^{2}\right\}}\right) \\
& =\frac{(k+2)^{2}}{(2 k+2)(2 k+3)}-\frac{k^{2}}{(2 k+1)(2 k+2)} \cdot \frac{2(2 k+1) x^{2}}{k^{2}\left\{(k+1)^{2}-2 k(k+2) x^{2}\right\}} \\
& =\frac{(k+1)\left\{(k+2)^{2}-2(k+1)(k+3) x^{2}\right\}}{2(2 k+3)\left\{(k+1)^{2}-2 k(k+2) x^{2}\right\}},
\end{aligned}
$$

which proves (8.1). Therefore $T_{x}$ is $k$-hyponormal $\Longleftrightarrow x \leq \frac{k+1}{\sqrt{2 k(k+2)}}$.

\section{ACKNOWLEDGEMENT}

The authors are very grateful to the referee for many helpful suggestions that improved the presentation. 


\section{REFERENCES}

[Bra] J. Bram, Subnormal operators, Duke Math. J. 22 (1955), 75-94. MR0068129 (16:835a)

[Con] J. B. Conway, The Theory of Subnormal Operators, Math. Surveys and Monographs vol. 36, Amer. Math. Soc., Providence, 1991. MR.1112128 (92h:47026)

[Cow1] C. Cowen, More subnormal Toeplitz operators, J. Reine Angew. Math. 367 (1986), 215-219. MR 0839133 (87h:47063)

[Cow2] Hyponormal and subnormal Toeplitz operators, Surveys of Some Recent Results in Operator Theory, I (J.B. Conway and B.B. Morrel, eds.), Pitman Research Notes in Mathematics, Vol 171, Longman, 1988, pp. 155-167. MR.0958573 (90j:47022)

[CoL] C. C. Cowen and J. J. Long, Some subnormal Toeplitz operators, J. Reine Angew. Math. 351 (1984), 216-220. MR0749683 (86h:47034)

[Cu1] R.E. Curto, Quadratically hyponormal weighted shifts, Integral Equations Operator Theory 13 (1990), 49-66. MR1025673 (90k:47061)

[Cu2] J Joint hyponormality: A bridge between hyponormality and subnormality, Operator Theory: Operator Algebras and Applications (Durham, NH, 1988) (W.B. Arveson and R.G. Douglas, eds.), Proc. Sympos. Pure Math., Vol. 51, part II, American Mathematical Society, Providence, (1990), Part 11, 69-91. MR.10774222 (91k:47049)

[CuF1] R.E. Curto and L.A. Fialkow, Recursiveness, positivity, and truncated moment problems, Houston J. Math. 17 (1991), 603-635. MR1147276 (93a:47016)

[CuF2] Recursively generated weighted shifts and the subnormal completion problem, Integral Equations Operator Theory 17 (1993), 202-2 46. MR1233668 (94h:47050)

[CuF3] - Recursively generated weighted shifts and the subnormal completion problem, II, Integral Equations Operator Theory 18 (1994), 36 9-426. MR 1265443 (94m:47044)

[CJP] R. E. Curto, I. B. Jung and S. S. Park, A characterization of k-hyponormality via weak subnormality, J. Math. Anal. Appl. 279 (2003), 556-568. MF 1974045 (2004b:47032)

[CLL] R. E. Curto, S. H. Lee and W. Y. Lee, Subnormality and 2-hyponormality for Toeplitz operators, Integral Equations Operator Theory 44 (2002), 138- 148. MR1930833 (2003f:47045)

[CuL1] R. E. Curto and W. Y. Lee, Joint hyponormality of Toeplitz pairs, Memoirs Amer. Math. Soc. no. 712, Amer. Math. Soc., Providence, 2001. MR.1810770|2002c:47042)

[CuL2] - Towards a model theory for 2-hyponormal operators, Integral Equations Operator Theory 44 (2002), 290-315. MR1933654 (2003m:47036)

[CuL3] Subnormality and k-hyponormality of Toeplitz operators: A brief survey and open questions, Operator theory and Banach algebras (Rabat, 1999), 73-81, Theta, Bucharest, 2003. MR2006315

[CMX] R. E. Curto, P. S. Muhly and J. Xia, Hyponormal pairs of commuting operators, Contributions to Operator Theory and Its Applications (Mesa, AZ, 1987) (I. Gohberg, J.W. Helton and L. Rodman, eds.), Operator Theory: Advances and Applications, vol. 35, Birkhäuser, Basel-Boston, (1988), 1-22. MR1017663 (90m:47037)

[DPY] R.G. Douglas, V.I. Paulsen, and K. Yan, Operator theory and algebraic geometry, Bull. Amer. Math. Soc. (N.S.) 20 (1989), 67-71. MR0955316 (90f:47028)

[Fan] P. Fan, Note on subnormal weighted shifts, Proc. Amer. Math. Soc. 103 (1988), 801802. MF 0947661 (89j:47016)

[Gu] C. Gu, Non-subnormal k-hyponormal Toeplitz operators, preprint, 2001.

[Ha1] P. R. Halmos, Ten problems in Hilbert space, Bull. Amer. Math. Soc. 76 (1970), 887933. MF $0270173(42: 5066)$

[Ha2] $\quad$ Ten years in Hilbert space, Integral Equations Operator Theory 2 (1979), 529-564. MR 0555777 (81c:47003)

[MaZ] J. Ma and S. Zhou, A necessary and sufficient condition for an operator to be subnormal, Nanjing Daxue Xuebao (Chinese) 2 (1985), 258-267. MR0834313 (87i:47031)

$[\mathrm{McCP}] \quad \mathrm{S}$. McCullough and V. Paulsen, A note on joint hyponormality, Proc. Amer. Math. Soc. 107 (1989), 187-195. MR0972236 (90a:47062)

[Smu] J. L. Smul'jan, An operator Hellinger integral (Russian), Mat. Sb. (N.S.) 91 (1959), 381-430. MR0121662(22:12396) 
[Spi] I. M. Spitkovskii, A criterion for normality of operators in Hilbert space, Funct. Anal. Appl. 16 (1982), 367-379. MR0659177|(83i:47033)

[Sta] J. G. Stampfli, Which weighted shifts are subnormal?, Pacific J. Math. 17 (1966), 367-379. MR 0193520(33:1740)

Department of Mathematics, University of Iowa, Iowa City, Iowa 52242

E-mail address: rcurto@math.uiowa.edu

Department of Mathematics, Seoul National University, Seoul 151-742, Korea

E-mail address: shlee@math.skku.ac.kr

Department of Mathematics, Seoul National University, Seoul 151-742, Korea

E-mail address: wylee@math.snu.ac.kr 